\title{
Study of dust deposition and temperature impact on solar photovoltaic module
}

\author{
Marek Jaszczur ${ }^{1, *}$, Qusay Hassan ${ }^{1}$, Janusz Teneta ${ }^{2}$, Katarzyna Styszko $^{1}$, Weronika Nawrot $^{1}$, and Robert Hanus ${ }^{3}$ \\ ${ }^{1}$ AGH University of Science and Technology, Faculty of Energy and Fuels, Poland \\ ${ }^{2}$ AGH University of Science and Technology, Faculty of Electrical Engineering, Automatics, Poland \\ ${ }^{3}$ Rzeszów University of Technology, Faculty of Electrical and Computer Engineering, 35-959 Rzeszów, Poland
}

\begin{abstract}
This work investigates the effect of the dust deposition and temperature on the photovoltaic module working under varying environmental conditions. The impact of the module temperature caused by the dust deposition, as well as direct impact caused by the dust particle absorption, scattering and reflectance on module performance, has been analysed. Presented results showed that dust deposition even in desert free country Poland influences the module temperature and power output significant. The estimated join effect of dust deposition and temperature increase, on power loss after three-four days of natural dust exposure in the city environment, was about 0.32 and 0.37 point of percentage for the selected sunny and cloudy days while evaluated PV system efficiency was in the range $13.37-14.14 \%$.
\end{abstract}

\section{Introduction}

Observed since 2011 the World energy demand growth rate is about $1.8 \%$ per year [1]. This continuous growth deteriorates the Earth ecological system and causes global warming phenomenon as well as rise $\mathrm{CO}_{2}$ emissions rate. The factors mentioned above motivate researchers to find alternative sources for conventional energy, in which the environmental aspect is taken into consideration. On the other hand the year 2016 has been recorded as the highest growth in the installation with the use of renewable energy [2].

Solar energy has been concerned as one of the most promising renewable energy resources with the World solar photovoltaic (PV) systems power cumulative capacity 301473 MW (in 2016) [3]. It is known that the performance of photovoltaic modules can be influenced by a large number of environmental and climatic factors such as incident irradiation, solar irradiation spectrum, temperature, wind speed, air pollution, tilt angle, snow and shadowing etc. [4].

One of the parameters that influence the energy conversion in PV modules, which can be a significant in many regions, is dust deposition. The dust particles deposited on the surface directly affect the transmittance of the solar collector, and photovoltaic modules lead to a substantial decrease in its performance [5]. There are number of studies in the literature which take into account the impact of the accumulative dust and environment conditions on the PV efficiency and energy production. Jamil et al. [6] presented a review of the research studies focusing on the effects of the environmental variables such as accumulative dust, wind speed, ambient temperature and humidity in addition to module tilt angle and orientation on the dust deposition on the PV modules. Mekhilef et al. [7] have done a similar review for effect of the dust, wind velocity, and relative humidity on the PV modules.

An experimental study carried out by Hassan et al. [8] was conducted under various environmental conditions in order to estimate PV modules energy conversion loss due to two independent effects: module operating temperature and natural dust deposition. A proposed new correlation allows to calculate the efficiency loss of the PV module caused by dust deposition, in addition to that authors obtained the module efficiency loss $4.6 \%$ and $5.8 \%$ at module temperature $30.9^{\circ} \mathrm{C}$ and $37.1^{\circ} \mathrm{C}$ respectively. Sarver et al. [9] prepared a review for the dust effect on PV performance for the different geographical region. Authors conclude that the duration of the dust deposition shows considerable variations depend on exposure time.

The module energy yield losses caused by dust deposition were studied by Sayyah et al. [10]. In that work authors concluded that the PV modules operate in different regions of the world, may require the additional cleaning processes including manual and automatic for assessing the most effective method for dust removing. The dust deposition accumulated on the PV modules surface depends upon two main factors: the local environmental conditions of the site (i.e. climate) and location with the orientation of the modules [11]. ElShobokshy and Hussein [12] illustrate the effect of the chemical and physical properties of the dust particles on the PV modules performance. In their investigation authors used artificial dust: limestone, carbon and cement with different diameters of $5,10,50,60$, and 80

\footnotetext{
*Corresponding author: jaszczur@agh.edu.pl
} 
$\mu \mathrm{m}$. The particles were deposited on the PV module front glass surface with a mass density of about $25 \mathrm{~g} / \mathrm{m}^{2}$. Authors conclude that the finer particles have a more degrading effect on cell performance than coarse particles.

The natural dust deposition on the PV modules front cover glass was experimentally analysed in [13] by means of several PV modules during exposure periods from 1 day up to 1 week. The results showed that dust deposition intensity on the module surface ranged from 7.5 to $42.1 \mathrm{mg} / \mathrm{m}^{2}$ for exposure periods of one day while the measured dust deposition for one week ranged from 25.8 to $277.0 \mathrm{mg} / \mathrm{m}^{2}$. The results also showed that the intensity and volume of precipitation in addition to the air humidity has a high impact on the dust particle deposition.

Kaldellis and Kokala [14] evaluated the effect of the air pollution on the photovoltaic module energy yield. The analysis was done based on the performance of five pairs of PV modules located on the building roof and operated in the urban environment of the city of Athens. The reported results showed that even a small amount of dust deposition about $1 \mathrm{~g} / \mathrm{m}^{2}$ could lead to energy losses corresponding almost $4 \%$ on an annual basis. Cabanillas and Munguia [15] in 20 days outdoor studies performed in city Hermosillo, Mexico investigate the dust effect on the PV module generated power. The authors showed that the dust deposition decreased the maximum power of a-Si, mono-Si, and poly-Si PV modules by $14 \%$, $8.5 \%$, and $5.2 \%$, respectively. The type of glass used on the front surface of the PV modules has a significant impact on the amount of accumulated dust.

Using the textured glass, module performance will increase due to a decrease in reflection losses at the same time this reduces the rate of dust accumulation [16]. The comparison of two different textured glass cover for the monocrystalline modules with $30^{\circ}$ tilted angle performed in order to the evaluated rate of accumulative dust was done by Piliougine et al. [17]. For the one year exposition, results show some difference in dust soiling between the textured glass and nontextured glass surfaces. In a study done in three different sites in the desert southern California, US by Caron and Littmann [18] investigate also PV modules energy losses due to the accumulative dust. The obtained maximum losses recorded with a fewer rainfall period were about $8.6 \%$. Based on the experimental work for PV energy losses due to soiling for three PV systems Kimber [19] validate a mathematical model for prediction energy losses.

It has been seen from the literature review that the dust deposition can significantly influence PV module performance. However, the studies were performed mainly for artificial dust or in the very dusty region. Most of the investigations presented in the literature were conducted in arid and semi-arid areas.

Only a few studies deal with natural dust. The key point which may be drawn from a review is that there is no theoretical or empirical model for PV module efficiency loss due to dust which is applicable to all the types of pollutants and different types of PV. Additionally, the models represent specific and particular cases of a narrow range of functionality. On the other hand, none of the work is taking into consideration at the same time effect of dust deposition and operating temperature and still it is not clear if deposited dust increases or decrease PV module operating temperature. For this reason, the objective of this work is to study - based on the experimental measurement - the impact of the temperature and natural dust deposition on the PV modules performance.

\section{Experimental and numerical set-up}

The experimental measurement for the dust deposition was conducted using two types of photovoltaic modules; the first type $(11$ pcs.) was polycrystalline BrukBet BEP260W module (temperature coefficient of power $0.40 \% /{ }^{\circ} \mathrm{C}$ and $1.62 \mathrm{~m}^{2}$ of surface area, white colour back sheet), the nominal module power is equal to $260 \mathrm{~W}$, and orientated at tilt angle $\beta=35^{\circ}$. The second type ( 2 pcs.) was a polycrystalline Sharp ND-RJ260 photovoltaic module (temperature coefficient of power $-0.42 \% /{ }^{\circ} \mathrm{C}$ and $1.6 \mathrm{~m}^{2}$ of surface area, white colour back sheet orientated at tilt angle $\beta=15^{\circ}$ ). The azimuth for all PV modules was the same $\gamma=20^{\circ}$ West and all modules were located in building C3 AGH University of Science and Technology in the city centre of Kraków, Poland $(50.066169 \mathrm{~N}, 19.921547 \mathrm{E})$.
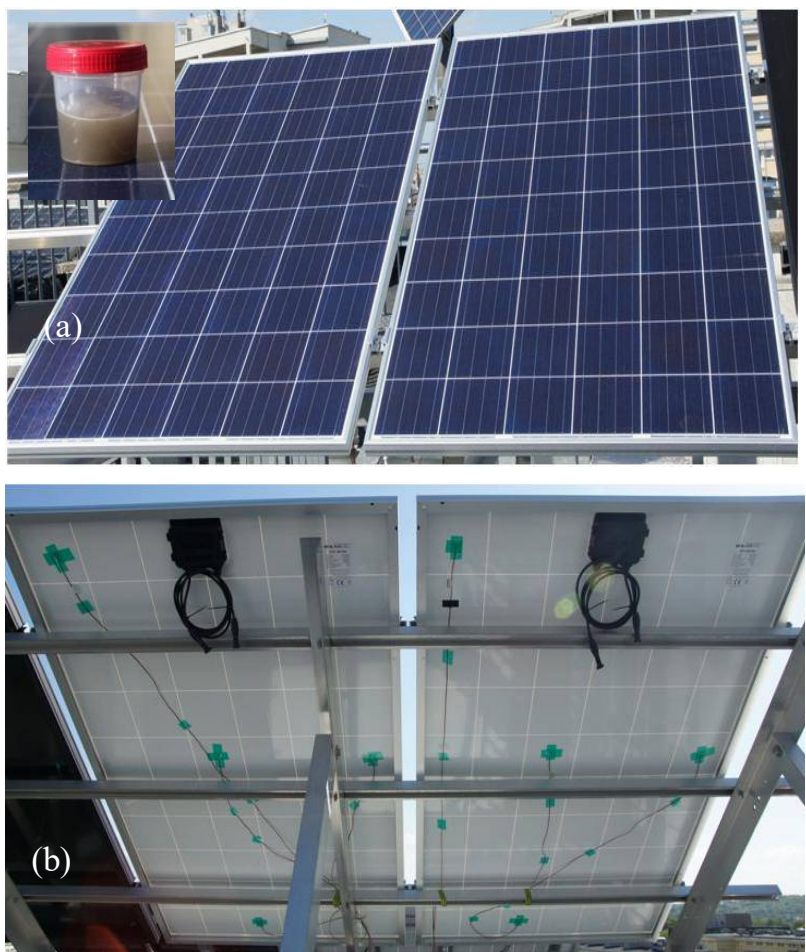

Fig. 1. The polycrystalline Sharp ND-RJ260 PV modules: front view with collected dust sample (a) and backside view with thermocouples arrangement (b).

The experimental set-up for the efficiency analysis consists of two described above Sharp ND-RJ260 photovoltaic modules connected to the Envertech EVT500 microinverter. The analysed modules electrical 
parameters (AC and DC) were acquired with 3-minutes temporal resolution using EnverBridge. The solar irradiation (global horizontal - GHI and diffuse horizontal - DHI components), ambient temperature Ta and other environmental factors were obtained from the local sensors (Pyranometers, Thermometer), at location AGH University of Science and Technology in the city centre of Kraków, Poland.

The main hardware components of the data logging system for temperature and solar irradiation measurement include four Advantech ADAM4018, 16bit, an 8-channel analogue input module that provides programmable input ranges on all channels, RS485 transceiver, computer unit with data acquisition software and storage. As a temperature sensors laboratory, calibrated (using certified 100-TP thermometer accuracy $\pm 0.02^{\circ} \mathrm{C}$ ) T-type thermocouples (Copper-Constantan) were used. Four thermocouples were attached to each analysed PV modules backsheet in the recommended by standard arrangement (see Fig. 1b).

The signals from all temperature sensors were acquired every second, and the average value was recorded every 60 seconds.For the present analysis of dust mass deposition, the naturally deposited dust was collected from all modules at morning hours at about 9 a.m. with different daily schedule in order to get different exposure period from 1 day up 7 days while for PV module performance analysis the dust from polycrystalline Sharp ND-RJ260 photovoltaic two modules were used olny. The right Sharp module was cleaned at the afternoons hours at 3:00 p.m., while the left (reference see Fig. 1a) module was cleaned at the morning hours at 9:00 a.m.

The deposited dust particles were removed using deionised water and a scratcher with suction pump [13]. The water with dust was collected in plastic containers, followed by freeze-drying (Liophilizator Alpha 1-4 LD). Dry mass of deposited dust was evaluated with laboratory class balance (OHAUS Discovery DV215CD). The PV module instantaneous power, $P_{P V}$ depends on the solar irradiance incident on the PV surface $G_{T}$, but it also depends on several additional factors such as dust and soiling, losses, shading, age, temperature and can be calculated from the following equation [20]:

$$
P_{P V}=\eta A_{c} G_{T}\left[1+\alpha_{P}\left(T_{c}-T_{C, S T C}\right)\right]
$$

where $\eta$ is the PV module efficiency, $G_{T}$ is the incident solar irradiation, $\alpha_{P}$ is the PV thermal coefficient of power, Tc is the PV module operating temperature, $T_{c, S T C}$ is the cell temperature at Standard Test Condition and $A_{C}$ is the module area. When incident solar irradiation $G_{T}$, module operating temperature $T_{c}$ and module power $P_{P V}$ are known from the measurement than eq. (1) can be used to calculate the PV module efficiency $\eta$ taking into account temperature effect or not as follows:

$$
\begin{gathered}
\eta_{p}=\frac{P_{P V}}{A_{C} G_{T}\left[1+\alpha_{P}\left(T_{C}-T_{C, S T C}\right)\right]} \\
\eta=\frac{P_{P V}}{A_{c} \cdot G_{T}}
\end{gathered}
$$

where $\eta_{p}$ is the PV module efficiency taking into account PV module operating temperature eq. (2) and $\eta$ is the PV efficiency without temperature consideration eq. (3)

\section{Results and discussion}

The dust samples were collected from all modules using the different exposure period from one day up to seven days for three consecutive weeks and were the subject of analysis. Dust deposition for modules with title angle $\beta=15^{\circ}, \beta=35^{\circ}$ are presented in Figures 2. Smaller title angle promotes substantial dust particle deposition.

Figure 3 shows the instantaneous solar irradiation global horizontal irradiation (GHI) and diffuse horizontal irradiation (DHI) components for two selected days: cloudy (04.06.2018) and sunny (07.06.2018) respectively. For the sunny day (Fig. 3a), dust particles were deposited for four days while for the cloudy day (Fig. 3b) exposition time was three days.
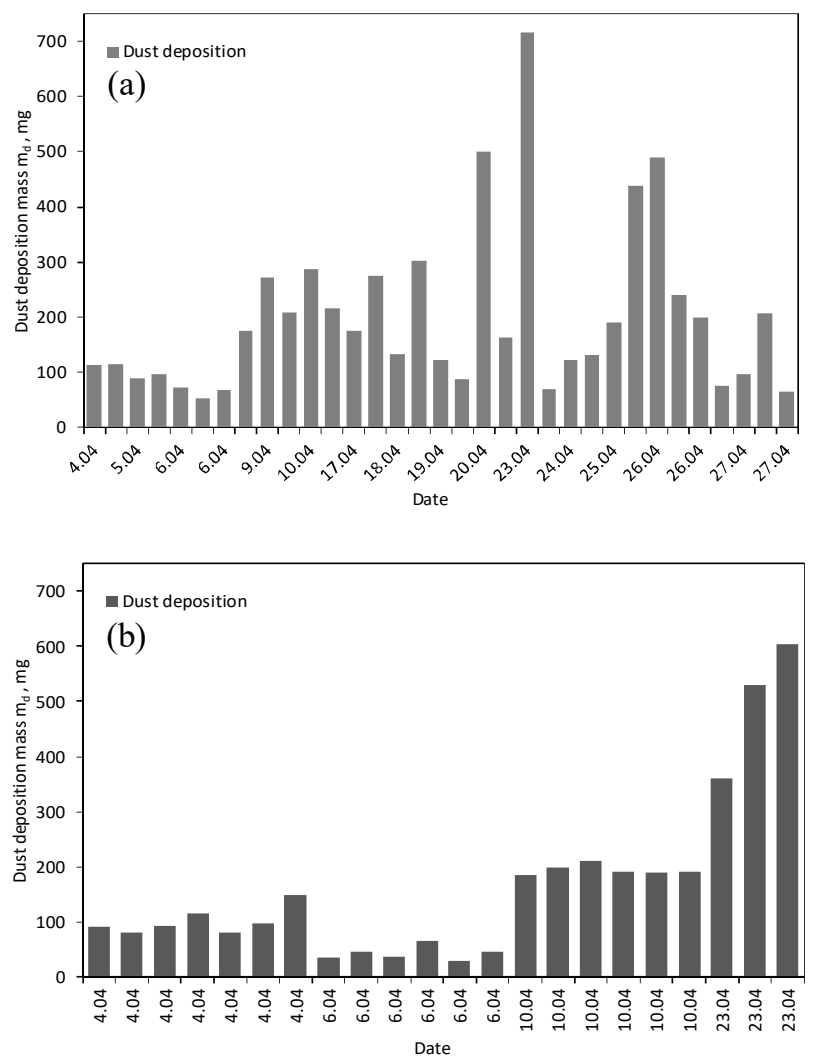

Fig. 2. Dust deposition on the PV module surface with a titled angle: $\beta=15^{\circ}$ (a) and $\beta=35^{\circ}(\mathrm{b})$. 

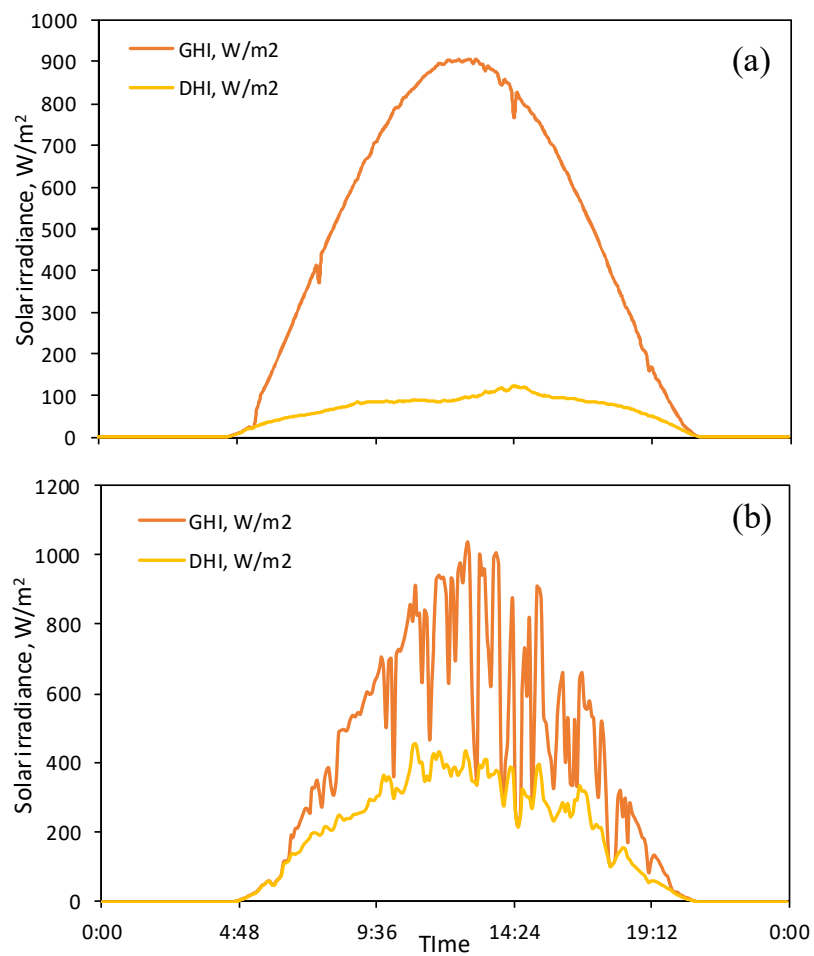

Fig. 3. Instantaneous solar irradiation components for: sunny (a) and cloudy (b) days.

Figure 4 show the instantaneous PV module temperature $T_{c}$ for clean $\mathrm{N} 1$ and dusty $\mathrm{N} 2$ module and for two selected days: cloudy and sunny respectively.
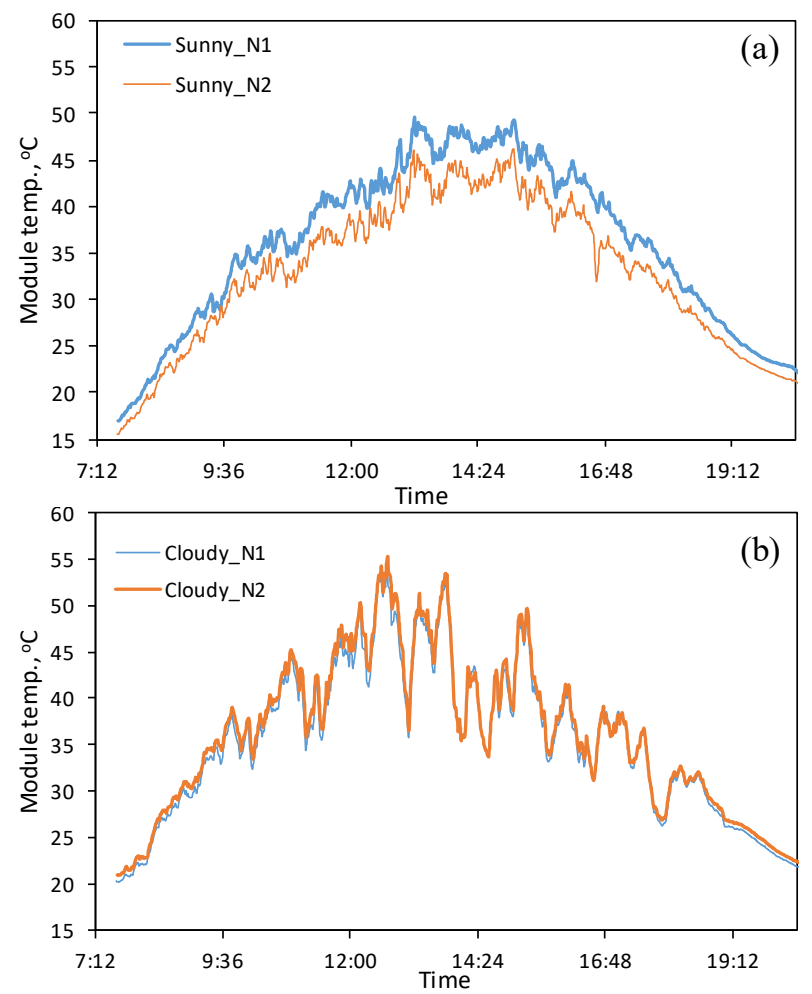

Fig. 4. PV module temperature $T_{c}$ for sunny (a) and cloudy (b) days.

In order to calculate PV module efficiency incident solar irradiation and electrical power from the PV system for a selected period is required. The PV system 10 minutes averaged AC electrical power is presented in Figures 5 for the cloudy (04.06.2018) and sunny (07.06.2018) days. The results show that the power generated by the system with the clean module is slightly higher than from unclean one.

In Figure 6 module efficiency $\eta$ is presented calculated with eq. (3). One may infer from this figures that the clean PV module efficiency $\eta$ is higher than from unclean one. This is observed for sunny (Fig. 6a) as well as (Fig. 6b) cloudy days. However, it is also worth to notice that for the sunny day almost continuous efficiency decreases during the day was observed. This is better seen when PV system power losses (absolute value in percentage points) or percentage power loss due to dust deposition and temperature for cloudy and sunny days respectively are calculated from as follows:

$$
\begin{array}{r}
\Delta_{\eta_{\text {loss }}}=\eta_{c}-\eta_{d} \\
\delta_{\eta_{\text {loss }}}=\frac{\eta_{c}-\eta_{d}}{\eta_{c}} \cdot 100 \%
\end{array}
$$

where $\eta_{c}, \eta_{d}$ represent the efficiency of the clean and dusty modules calculated using the eq. (2) or eq. (3) for the clean or dusty modules data. In Figure 7 photovoltaic module power losses are presented. For sunny day an increase in the power losses is observed up to 2 p.m. For cloudy day continuous increase is seen, however, at 11:00 a.m. negative efficiency loss is observed.
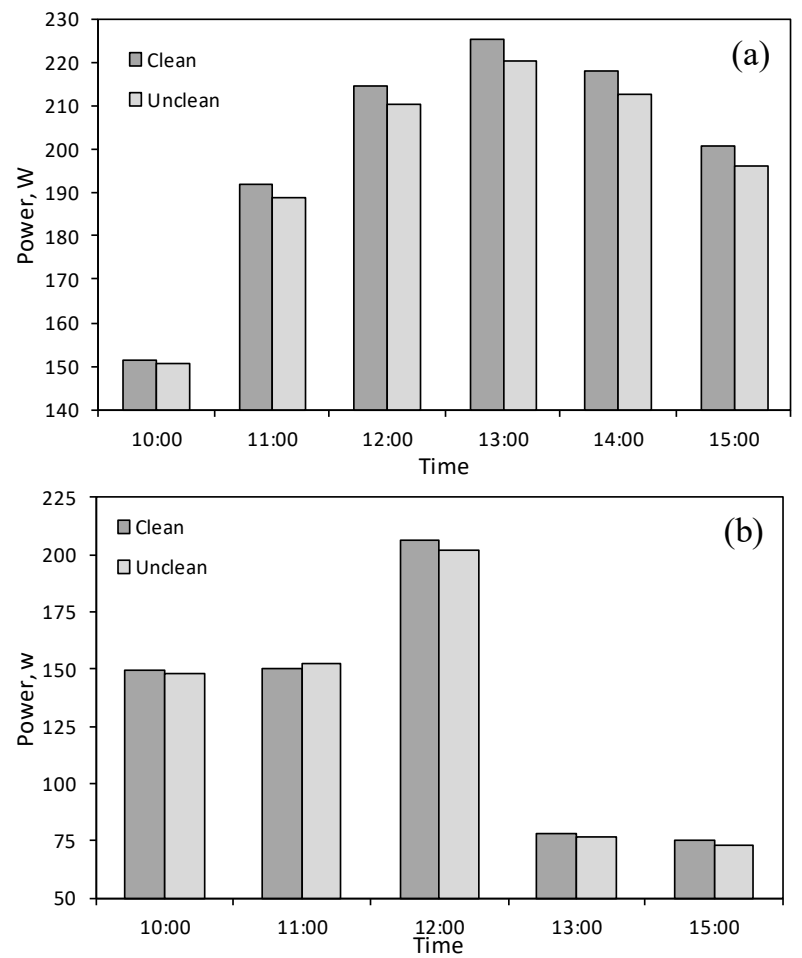

Fig. 5. Photovoltaic module 10-minutes averaged electrical power for: sunny (a) and cloudy (b) days.

The effect is due to very high solar irradiation fluctuation which creates difficulties for accurate 
maximum power point (MPPT) measurement simultaneously at two different analysed photovoltaic modules. The observed maximum efficiency losses $\Delta_{\eta_{\text {loss }}}$ are about 0.32 and 0.37 point of percentage for selected sunny and cloudy day respectively.
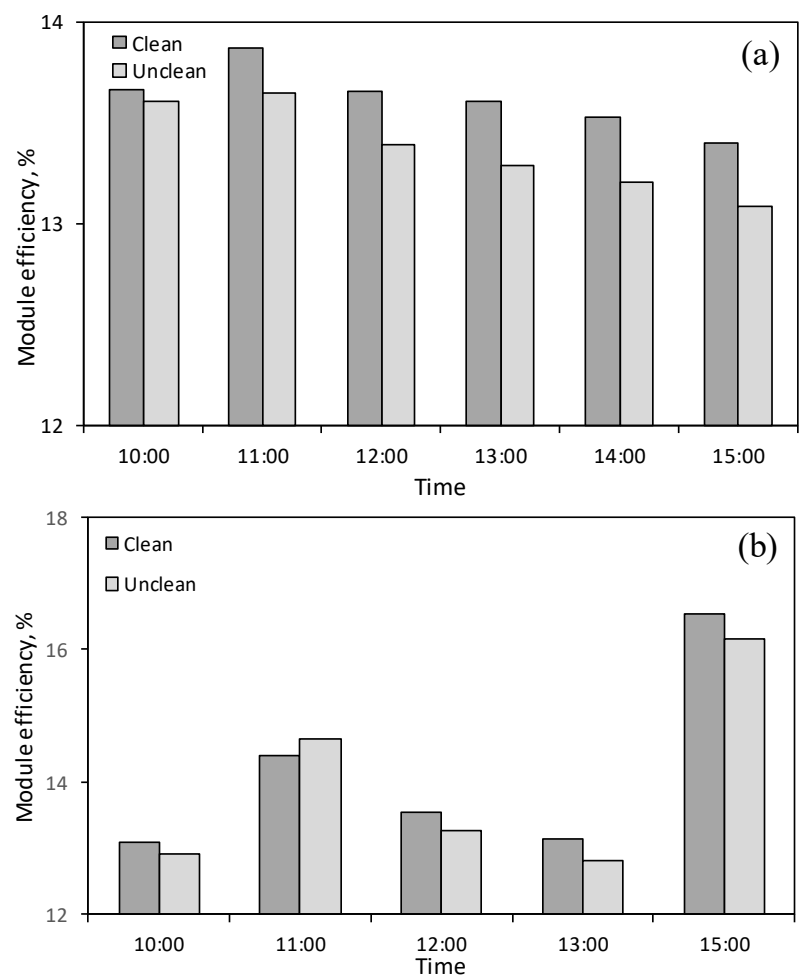

Fig. 6. Photovoltaic module 10-minutes averaged efficiency at a selected time and for: sunny (a) and cloudy (b) days.
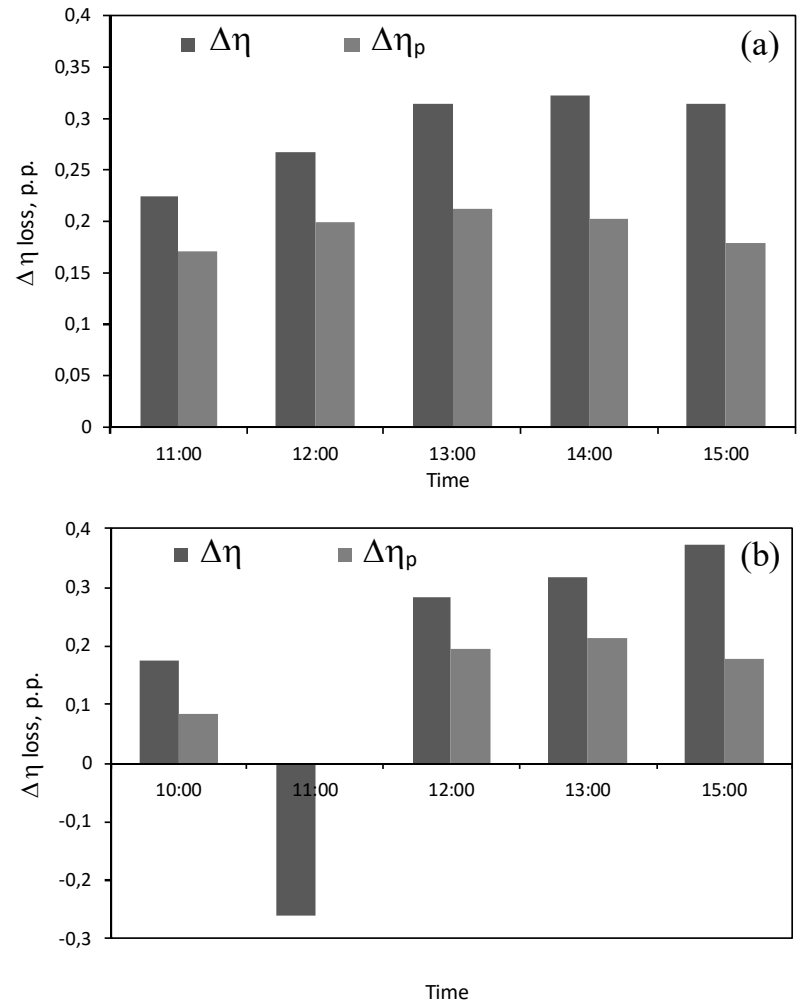

Fig. 7. Photovoltaic module efficiency loss (in percentage points) at a selected time and for: sunny (a) and cloudy (b) days
In Figure 7 two different efficiency losses are presented. The first one is the total efficiency loss $\Delta \eta$ calculated with eq. $(3,4)$ and it is related to dust deposition and to PV module operating temperature difference (in reference to the clean module) caused by the dust deposition.

While the second efficiency loss $\Delta \eta_{\mathrm{p}}$ is the efficiency loss which takes into account the only direct dust deposition effect excluding temperature increase due to dust at the module surface. In that case, the efficiency of the clean and the dusty modules are calculated from the eq. $(2,4)$ and takes into account the clean and unclean module operating temperature $T_{c}$.

One may infer from Figures 7 that efficiency loss excluding dust-temperature related phenomena is 24$43 \%$ lower. This confirmed that direct dust deposition effect which causes reduction of solar irradiation intensity that can reach the photovoltaic module surfaces has a dominant effect. In another word absorption, scattering and reflectance of the incident solar radiation due to particle layer at the PV module from surface plays a more important role than dust related indirect thermal effect (module temperature increase due to dust particle deposition), however, the secondary effect for the presented cases is not negligible.

\section{Conclusions}

This study investigates the impact of dust deposition and the operating temperature on the photovoltaic module performance working under varying environmental conditions.

The focus is on the evaluation of the PV modules energy conversion loss due to two effects: one related to absorption, scattering and reflectance of the incident solar radiation due to dust layer at the PV module, and the second one related to the indirect thermal effect module temperature change due to dust particle deposition.

In the experimental study conducted under variable environmental conditions, it was seen that the PV module power output is influenced by the dust deposited particles and the effect is non-negligible even for as short as three-four days of natural dust deposition in desert free country Poland.

The maximum observed efficiency loss equal to 0.32 and 0.37 point of percentage (which results in the power losses of about $2.39 \%$ and $2.26 \%$ see eq. (5)) and was observed for presented sunny and cloudy days. Results shows that efficiency loss excluding dust-temperature related phenomena is $24-43 \%$ lower which confirmed that direct dust deposition effect has a dominant effect.

On the basis of the presented analysis, one may state that the particles which exist in the air of urban areas negatively affect the PV module efficiency and the level of power losses is non-negligible. The efficiency loss gradually increased with the dust deposited mass which was presented in another authors work [5] together with the mathematical model of this phenomena. 


\section{Acknowledgements}

The photovoltaic installation was funded by the National Fund for Environmental Protection and Water Management, Grant 139/2015/Wn06/OA-is-ku/D. This work was partially financed by AGH University Grant No.11.11.210.312 and No. 15.11.210.436.

\section{References}

1. L. Sawin at al. Renewables, Global Status Report, (2017)

2. Renewable energy Mid-Term market report, https://www.iea.org, (2016)

3. British Petroleum Statistical Review of World Energy, http://www.bp.com/statisticalreview, (2017)

4. Q. Hassan, M. Jaszczur, E. Przenzak, J. Abdulateef, Contemporary Problems of Power Engineering and Environmental Protection, 33. (2016)

5. M. Jaszczur, J. Teneta, K. Styszko, Q. Hassan, P. Burzyńska, E. Marcinek, N. Łopian, Environmental Science and Pollution Research, 1-16. (2018)

6. J. Jamil, A. Rahman, S. Shaari, Renewable and Sustainable Energy Reviews 67 (2017)

7. S. Mekhilef, R. Saidur, M. Kamalisarvestani, Renewable and Sustainable Energy Reviews. 16 (2012)

8. Q. Hassan, M. Jaszczur, K. Styszko, J. Teneta, M. Dudek, Thermal Science Journal (2018)

9. T. Sarver, A. Qaraghuli, L. A. Kazmerski, Renewable and Sustainable Energy Reviews. 22, (2013)

10. A. Sayyah, N. Horenstein, K. Mazumder, Solar Energy 107, 576 (2014)

11. M. Mani, R. Pillai, Renewable and Sustainable Energy Reviews 14,3124 (2010)

12. S. El-Shobokshy, M. Hussein,.Solar energy 51, 505 (1993)

13. K. Styszko, M. Jaszczur, J. Teneta, Q. Hassan, P. Burzyńska, E. Marcinek, L. Samek, Environmental Science and Pollution Research, 1 (2018)

14. K. Kaldellis, A. Kokala, Energy, 35, 4862 (2010)

15. E. Cabanillas, H. Munguía, Journal of Renewable and Sustainable Energy, 3, 043114 (2011)

16. A. Nositschka, D. Neumann, O. Prast, F. Gromball, In 22nd European Photovoltaic Solar Energy Conference (2007)

17. M. Piliougine, J. Carretero, M. Sidrach, D. Montiel, P. Sánchez-Friera, In Proceedings of 23rd European Solar Energy Conference (2008)

18. R. Caron, B. Littmann, IEEE Journal of Photovoltaics, 3, (2013)

19. A. Kimber, In Proceedings $22^{\text {nd }}$ European Photovoltaic Solar Energy Conference (2007)

20. Q. Hassan, M. Jaszczur, E. Przenzak, In E3S Web of Conferences (Vol. 14, p. 01028). EDP Sciences, 14, 01028 (2017) 